\title{
Cationic Palladium Bis-carbene Carboxylate Complexes
}

\author{
J. Chris Slootweg, and Peter Chen*
}

Supporting Information

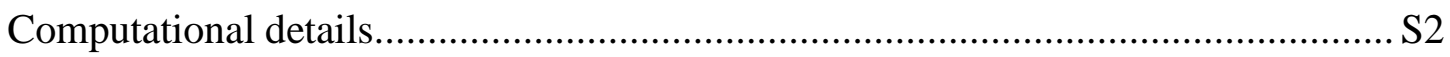

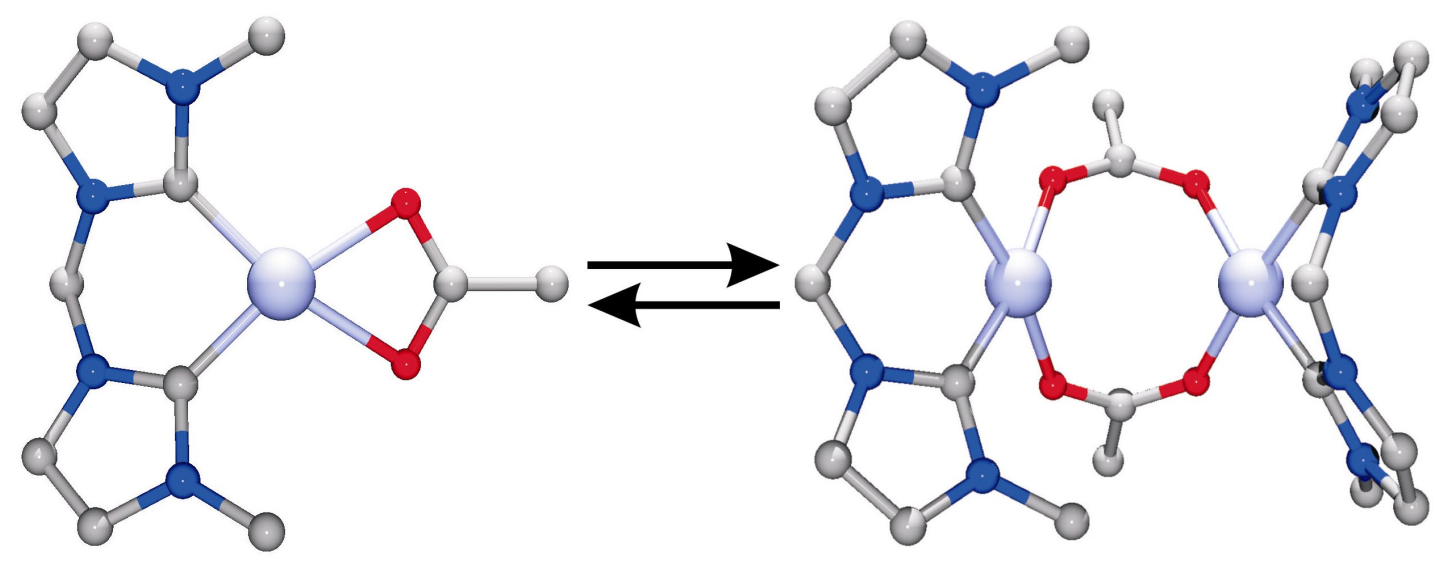

All Cartesian coordinates are given in Angstroms 

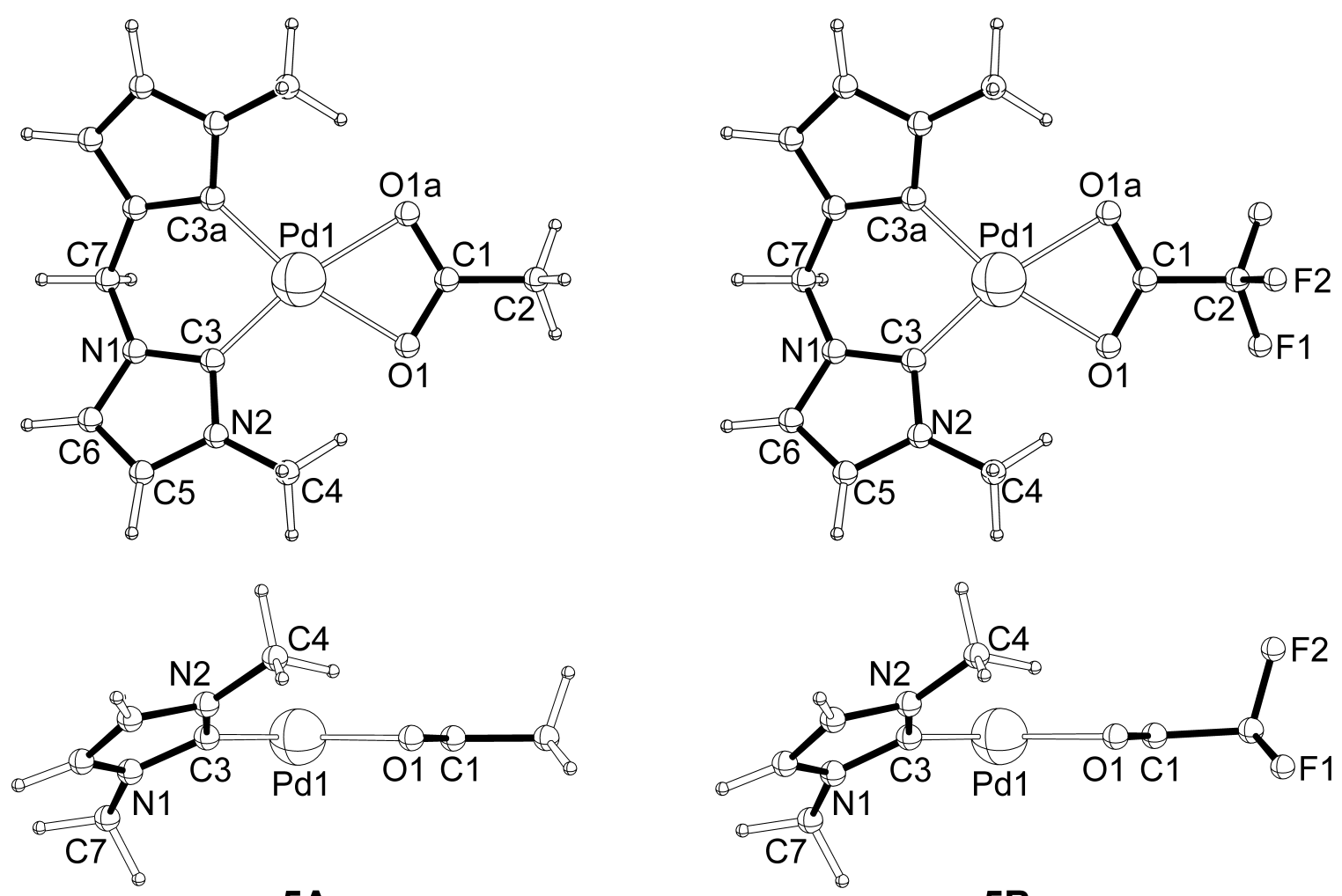

5A

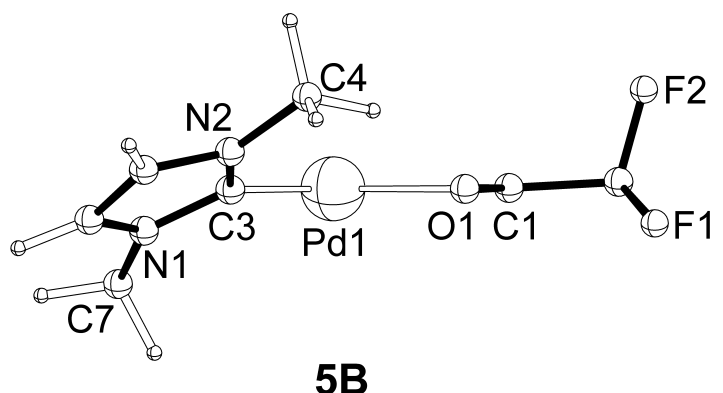

Figure 1. Calculated structures of (1,1'-dimethyl-3,3'-methylenediimidazol-2,2'-diylidene) $\operatorname{Pd}\left(\kappa^{2} O, O^{\prime}\right.$ $\left.\mathrm{OCOCH}_{3}\right)^{+}(\mathbf{5 A})$ and $\left(1,1^{\prime}\right.$-dimethyl-3,3'-methylenediimidazol-2,2'-diylidene $) \operatorname{Pd}\left(\kappa^{2} O, O^{\prime}-\mathrm{OCOCF}_{3}\right)^{+}(\mathbf{5 B})$ (both $C_{s}$ symmetry) at B3PW91/6-31+G** (LANL2DZ for Pd). Selected bond lengths $[\AA]$ and angles $\left[{ }^{\circ}\right]$ for 5A [5B]: Pd1-O1 2.123 [2.158], Pd1-C3 1.978 [1.971], O1-C1 1.279 [1.264], C1-C2 1.491 [1.543], C3-N1 1.355 [1.355], C3-N2 1.348 [1.347], C4-N2 1.462 [1.464], C7-N1 1.451 [1.450]; O1-Pd1-O1a 62.04 [61.43], O1-Pd1-C3a 167.98 [167.80], C3-Pd1-C3a 86.06 [85.82], N1-C3-N2 105.42 [105.61]. 

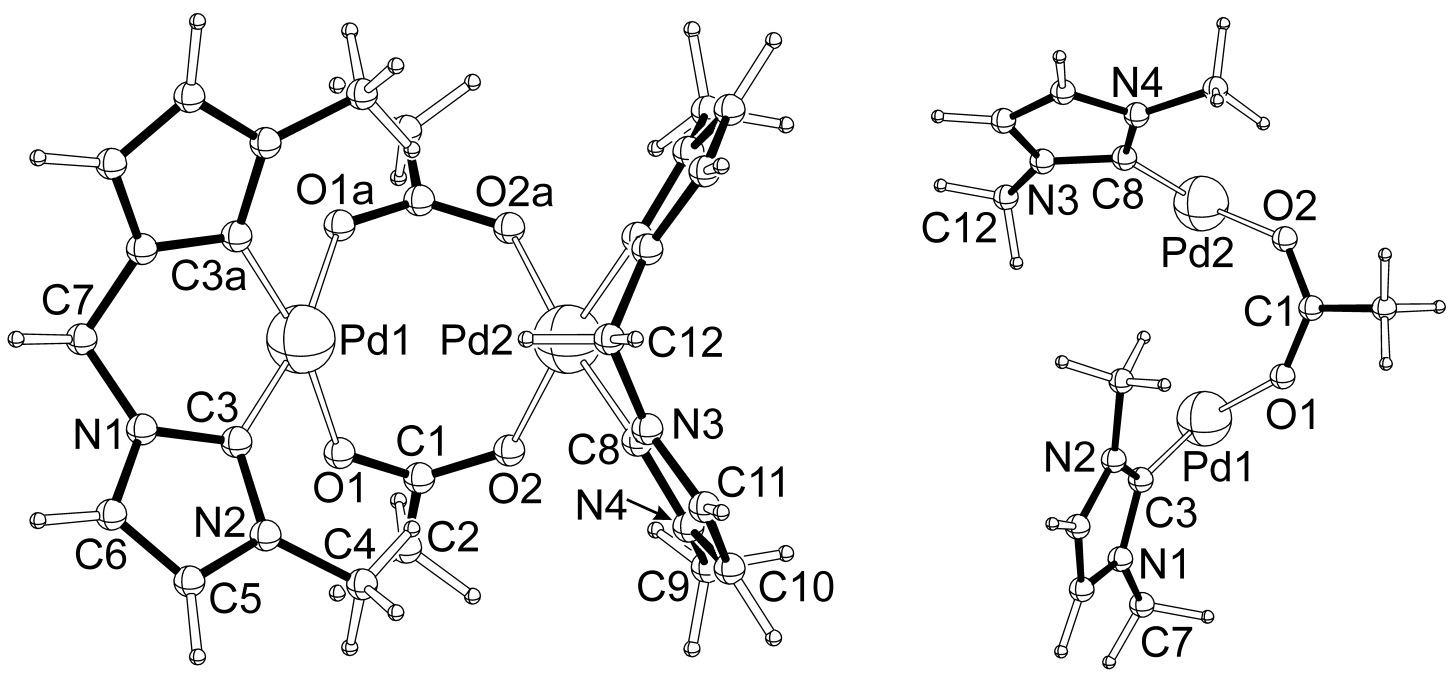

$(5 \mathrm{~A})_{2}$

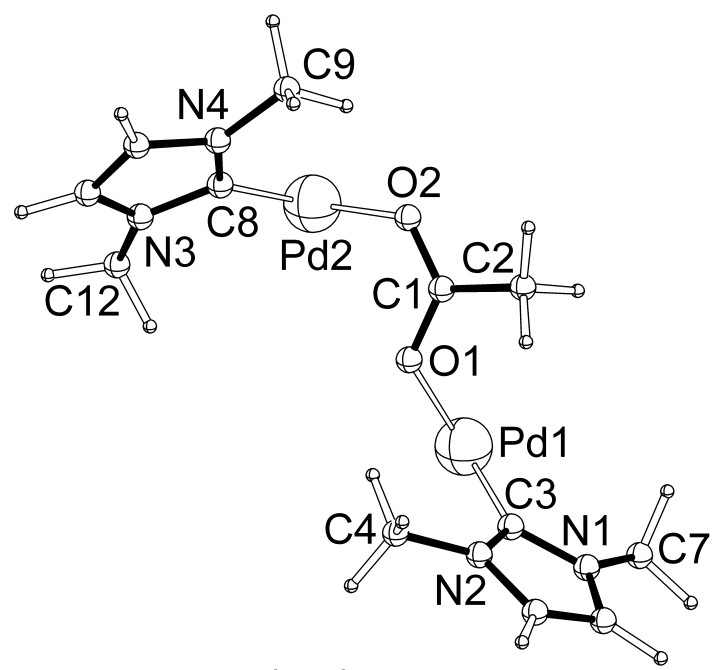

$(5 \mathrm{~A})_{2}$ anti

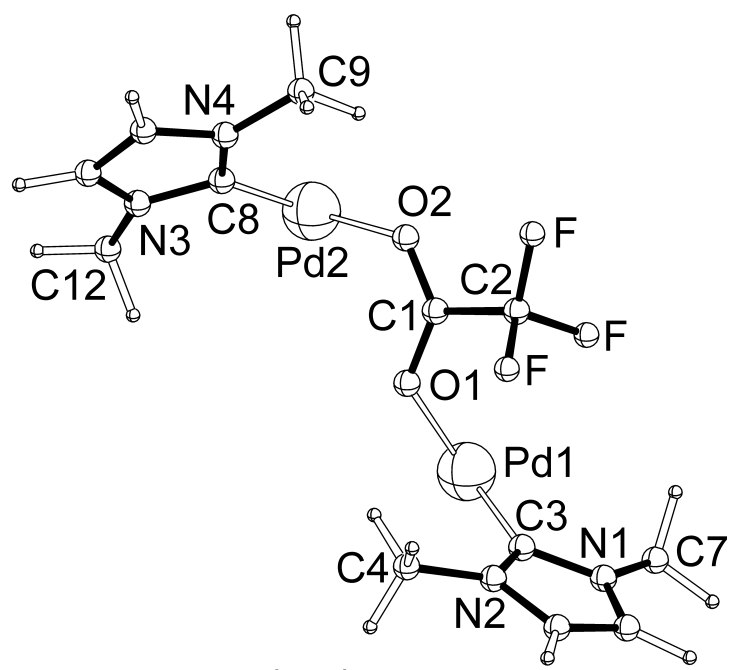

$(5 \mathrm{~B})_{2}$ anti

Figure 2. Calculated structures of $(\mathbf{5 A})_{2},(\mathbf{5 A})_{2} \_a n t i$, and $(\mathbf{5 B})_{2 \_}$anti (all $C_{s}$ symmetry) at B3PW91/6$31+\mathrm{G}^{* *}$ (LANL2DZ for Pd). Selected bond lengths $[\AA]$ and angles $\left[{ }^{\circ}\right]$ for $(\mathbf{5 A})_{2}\left[(\mathbf{5 A})_{2 \_}\right.$anti]: Pd1-Pd2 3.541 [4.479], Pd1-O1 2.092 [2.114], Pd2-O2 2.090 [2.117], Pd1-C3 1.986 [1.977], Pd2-C8 1.990 [1.983], O1-C1 1.264 [1.274], O2-C1 1.269 [1.264], C1-C2 1.509 [1.510], C3-N1 1.356 [1.356], C3-N2 1.348 [1.347], C4-N2 1.456 [1.462], C7-N1 1.451 [1.452], C8-N3 1.358 [1.356], C8-N4 1.348 [1.347], C9-N4 1.464 [1.462], C12-N3 1.452 [1.452]; O1-Pd1-O1a 91.60 [81.35], O2-Pd2-O2a 89.10 [88.18], O1-Pd1-C3a 168.75 [177.92], O2-Pd2-C8a 174.04 [176.86], C3-Pd1-C3a 85.95 [85.19], C8-Pd2-C8a 84.40 [84.86], N1-C3-N2 105.15 [105.35], N3-C8-N4 105.23 [105.32]. (5B)2_anti: Pd1-O1 2.147, Pd2O2 2.127, Pd1-C3 1.969, Pd2-C8 1.979, O1-C1 1.256, O2-C1 1.252, C1-C2 1.556, C3-N1 1.355, C3-N2 1.345, C4-N2 1.461, C7-N1 1.454, C8-N3 1.357, C8-N4 1.346, C9-N4 1.463, C12-N3 1.453; O1-Pd1O1a 80.34, O2-Pd2-O2a 89.07, O1-Pd1-C3a 177.31, O2-Pd2-C8a 176.92, C3-Pd1-C3a 84.52, C8-Pd2C8a 84.59, N1-C3-N2 105.75, N3-C8-N4 105.54. 
Table 1. Absolute energies (a.u.)

\begin{tabular}{c|c} 
& Energies \\
\hline $\mathbf{5 A}$ & -924.0287833 \\
\hline $\mathbf{5 B}$ & -1221.6285989 \\
\hline$(\mathbf{5 A})_{2}$ & -1848.0138403 \\
\hline$(\mathbf{5 A})_{2}$ anti & -1848.0164374 \\
\hline$(\mathbf{5 B})_{2}$ & not a minimum \\
\hline$(\mathbf{5 B})_{2 \_a n t i}$ & -2443.2355533
\end{tabular}

5A $\left(C_{s}\right)$

\begin{tabular}{llll} 
atom & \multicolumn{1}{l}{ X } & \multicolumn{1}{l}{ Y } \\
$\mathrm{H}$ & -2.259346 & 0.283086 & 3.180615 \\
$\mathrm{H}$ & -2.259346 & 0.283086 & -3.180615 \\
$\mathrm{C}$ & -1.173920 & 0.406998 & -3.205398 \\
$\mathrm{C}$ & -1.173920 & 0.406998 & 3.205398 \\
$\mathrm{H}$ & -0.536778 & 5.039551 & 0.000000 \\
$\mathrm{H}$ & -0.759507 & -2.164131 & -4.212976 \\
$\mathrm{H}$ & -0.759507 & -2.164131 & 4.212976 \\
$\mathrm{H}$ & -0.886868 & 1.304285 & -2.658014 \\
$\mathrm{H}$ & -0.886868 & 1.304285 & 2.658014 \\
$\mathrm{H}$ & -0.837368 & 0.494579 & 4.239921 \\
$\mathrm{H}$ & -0.837368 & 0.494579 & -4.239921 \\
$\mathrm{C}$ & -0.403479 & -1.985411 & 3.209899 \\
$\mathrm{C}$ & -0.403479 & -1.985411 & -3.209899 \\
$\mathrm{~N}$ & -0.527844 & -0.752869 & -2.592847 \\
$\mathrm{~N}$ & -0.527844 & -0.752869 & 2.592847 \\
$\mathrm{C}$ & 0.207564 & -2.817451 & -2.328497 \\
$\mathrm{C}$ & 0.207564 & -2.817451 & 2.328497 \\
$\mathrm{C}$ & 0.468528 & 4.603368 & 0.000000 \\
$\mathrm{C}$ & -0.008037 & -0.794822 & -1.349891 \\
$\mathrm{C}$ & -0.008037 & -0.794822 & 1.349891 \\
$\mathrm{H}$ & 0.498482 & -3.853006 & 2.416805 \\
$\mathrm{H}$ & 0.498482 & -3.853006 & -2.416805 \\
$\mathrm{C}$ & 0.347894 & 3.117192 & 0.000000 \\
$\mathrm{O}$ & 0.284951 & 2.457077 & 1.094448 \\
$\mathrm{O}$ & 0.284951 & 2.457077 & -1.094448 \\
$\mathrm{Pd}$ & 0.112774 & 0.646081 & 0.000000 \\
$\mathrm{~N}$ & 0.436058 & -2.065359 & 1.188813 \\
$\mathrm{~N}$ & 0.436058 & -2.065359 & -1.188813 \\
$\mathrm{H}$ & 0.986887 & 4.942218 & -0.898645 \\
$\mathrm{H}$ & 0.986887 & 4.942218 & 0.898645 \\
$\mathrm{H}$ & 1.203916 & -3.588454 & 0.000000
\end{tabular}




$\begin{array}{llll}\mathrm{C} & 1.144447 & -2.499503 & 0.000000 \\ \mathrm{H} & 2.155755 & -2.081062 & 0.000000\end{array}$

\begin{tabular}{lllr} 
SB & $\left(\boldsymbol{C}_{\boldsymbol{s}}\right)$ & & \\
atom & $\mathrm{X}$ & $\mathrm{Y}$ & \multicolumn{1}{l}{} \\
$\mathrm{H}$ & 2.216103 & -2.639676 & 0.000000 \\
$\mathrm{H}$ & 1.314393 & -4.177712 & 0.000000 \\
$\mathrm{C}$ & 1.219203 & -3.091377 & 0.000000 \\
$\mathrm{~F}$ & 0.831936 & 4.495087 & 1.087100 \\
$\mathrm{~F}$ & 0.831936 & 4.495087 & -1.087100 \\
$\mathrm{H}$ & 0.629444 & -4.456778 & 2.425532 \\
$\mathrm{H}$ & 0.629444 & -4.456778 & -2.425532 \\
$\mathrm{~N}$ & 0.493751 & -2.680758 & 1.187457 \\
$\mathrm{~N}$ & 0.493751 & -2.680758 & -1.187457 \\
$\mathrm{C}$ & 0.292691 & -3.435814 & -2.330044 \\
$\mathrm{C}$ & 0.292691 & -3.435814 & 2.330044 \\
$\mathrm{C}$ & 0.218893 & 4.023668 & 0.000000 \\
$\mathrm{C}$ & 0.209299 & 2.480648 & 0.000000 \\
$\mathrm{O}$ & 0.184328 & 1.860891 & 1.101874 \\
$\mathrm{O}$ & 0.184328 & 1.860891 & -1.101874 \\
$\mathrm{Pd}$ & 0.068578 & 0.009605 & 0.000000 \\
$\mathrm{C}$ & -0.007480 & -1.432145 & -1.342312 \\
$\mathrm{C}$ & -0.007480 & -1.432145 & 1.342312 \\
$\mathrm{C}$ & -0.359351 & -2.627137 & 3.204093 \\
$\mathrm{C}$ & -0.359351 & -2.627137 & -3.204093 \\
$\mathrm{~N}$ & -0.536407 & -1.404350 & -2.580888 \\
$\mathrm{~N}$ & -0.536407 & -1.404350 & 2.580888 \\
$\mathrm{H}$ & -0.918254 & -0.171549 & 4.224110 \\
$\mathrm{H}$ & -0.918254 & -0.171549 & -4.224110 \\
$\mathrm{H}$ & -0.711925 & -2.816112 & -4.206583 \\
$\mathrm{H}$ & -0.711925 & -2.816112 & 4.206583 \\
$\mathrm{H}$ & -0.992587 & 0.636729 & -2.644999 \\
$\mathrm{H}$ & -0.992587 & 0.636729 & 2.644999 \\
$\mathrm{~F}$ & -1.053604 & 4.461379 & 0.000000 \\
$\mathrm{C}$ & -1.241552 & -0.274515 & 3.187015 \\
$\mathrm{H}$ & -1.241552 & -0.274515 & -3.187015 \\
$\mathrm{H}$ & -2.319501 & -0.449032 & 3.151855 \\
$\mathrm{H}$ & -2.319501 & -0.449032 & -3.151855
\end{tabular}

$(5 \mathrm{~A})_{2} \quad\left(C_{s}\right)$

atom $X$ 


\begin{tabular}{|c|c|c|c|}
\hline C & -2.342634 & -0.054072 & 1.830304 \\
\hline O & -1.859547 & 1.066902 & 1.500183 \\
\hline C & -2.342634 & -0.054072 & -1.830304 \\
\hline O & -1.922037 & -1.195434 & 1.465808 \\
\hline O & -1.859547 & 1.066902 & -1.500183 \\
\hline O & -1.922037 & -1.195434 & -1.465808 \\
\hline $\mathrm{Pd}$ & -0.570337 & 1.747568 & 0.000000 \\
\hline $\mathrm{H}$ & -0.586266 & 5.007170 & 0.000000 \\
\hline Pd & -0.558159 & -1.792972 & 0.000000 \\
\hline C & 0.490954 & 2.740999 & 1.353831 \\
\hline $\mathrm{N}$ & 0.936284 & 2.412479 & 2.582940 \\
\hline C & 0.487648 & 4.797794 & 0.000000 \\
\hline $\mathrm{N}$ & 0.840212 & 4.040994 & 1.187243 \\
\hline $\mathrm{N}$ & 0.498092 & -3.260766 & 2.467685 \\
\hline C & 1.543584 & 3.501432 & 3.187976 \\
\hline C & 0.490954 & 2.740999 & -1.353831 \\
\hline C & 1.485417 & 4.532321 & 2.308654 \\
\hline C & 0.702977 & -2.557088 & 1.336647 \\
\hline $\mathrm{N}$ & 0.840212 & 4.040994 & -1.187243 \\
\hline $\mathrm{N}$ & 0.936284 & 2.412479 & -2.582940 \\
\hline $\mathrm{H}$ & 1.039933 & 5.737931 & 0.000000 \\
\hline C & 0.702977 & -2.557088 & -1.336647 \\
\hline $\mathrm{N}$ & 0.498092 & -3.260766 & -2.467685 \\
\hline C & 1.707480 & -3.651133 & 3.020264 \\
\hline C & 1.485417 & 4.532321 & -2.308654 \\
\hline C & 1.543584 & 3.501432 & -3.187976 \\
\hline $\mathrm{N}$ & 2.051842 & -2.513788 & 1.182771 \\
\hline $\mathrm{H}$ & 2.492790 & -0.848437 & 0.000000 \\
\hline C & 2.692174 & -3.187621 & 2.209296 \\
\hline $\mathrm{N}$ & 2.051842 & -2.513788 & -1.182771 \\
\hline C & 2.656924 & -1.929197 & 0.000000 \\
\hline C & 1.707480 & -3.651133 & -3.020264 \\
\hline C & 2.692174 & -3.187621 & -2.209296 \\
\hline $\mathrm{H}$ & 3.727115 & -2.137284 & 0.000000 \\
\hline $\mathrm{H}$ & 1.761666 & -4.227796 & 3.931503 \\
\hline $\mathrm{H}$ & 3.766190 & -3.271435 & 2.279776 \\
\hline $\mathrm{H}$ & 3.766190 & -3.271435 & -2.279776 \\
\hline $\mathrm{H}$ & 1.761666 & -4.227796 & -3.931503 \\
\hline $\mathrm{H}$ & 1.963667 & 3.448286 & 4.180941 \\
\hline $\mathrm{H}$ & 1.835976 & 5.550351 & 2.386713 \\
\hline $\mathrm{H}$ & 1.835976 & 5.550351 & -2.386713 \\
\hline
\end{tabular}




$\begin{array}{lrrr}\mathrm{H} & 1.963667 & 3.448286 & -4.180941 \\ \mathrm{C} & 0.799634 & 1.113111 & -3.227373 \\ \mathrm{H} & 0.420439 & 0.400528 & -2.497447 \\ \mathrm{H} & 0.096045 & 1.184771 & -4.059900 \\ \mathrm{H} & 1.774910 & 0.790207 & -3.598883 \\ \mathrm{C} & 0.799634 & 1.113111 & 3.227373 \\ \mathrm{H} & 0.096045 & 1.184771 & 4.059900 \\ \mathrm{H} & 0.420439 & 0.400528 & 2.497447 \\ \mathrm{H} & 1.774910 & 0.790207 & 3.598883 \\ \mathrm{C} & -0.799692 & -3.674310 & 3.003707 \\ \mathrm{H} & -0.821453 & -3.481136 & 4.077995 \\ \mathrm{H} & -1.581534 & -3.096887 & 2.513926 \\ \mathrm{H} & -0.943845 & -4.742242 & 2.823131 \\ \mathrm{C} & -0.799692 & -3.674310 & -3.003707 \\ \mathrm{H} & -1.581534 & -3.096887 & -2.513926 \\ \mathrm{H} & -0.821453 & -3.481136 & -4.077995 \\ \mathrm{H} & -0.943845 & -4.742242 & -2.823131 \\ \mathrm{C} & -3.539733 & -0.034980 & -2.748060 \\ \mathrm{H} & -4.444355 & -0.049589 & -2.130087 \\ \mathrm{H} & -3.555821 & 0.877101 & -3.345615 \\ \mathrm{H} & -3.560295 & -0.919479 & -3.386464 \\ \mathrm{H} & -3.539733 & -0.034980 & 2.748060 \\ \mathrm{H} & -3.555821 & 0.877101 & 3.345615 \\ \mathrm{H} & -4.444355 & -0.049589 & 2.130087 \\ \mathrm{H} & -3.560295 & -0.919479 & 3.386464\end{array}$

\begin{tabular}{|c|c|c|c|}
\hline atom & $x$ & $y$ & $z$ \\
\hline C & -1.280302 & 0.018575 & 1.744479 \\
\hline O & -0.167640 & 0.521106 & 1.378115 \\
\hline C & -1.280302 & 0.018575 & -1.744479 \\
\hline O & -1.614492 & -1.169502 & 1.472761 \\
\hline O & -0.167640 & 0.521106 & -1.378115 \\
\hline O & -1.614492 & -1.169502 & -1.472761 \\
\hline $\mathrm{Pd}$ & 0.087866 & 2.103625 & 0.000000 \\
\hline $\mathrm{H}$ & -1.854902 & 4.725186 & 0.000000 \\
\hline $\mathrm{Pd}$ & -0.617813 & -2.319036 & 0.000000 \\
\hline C & 0.347040 & 3.536384 & 1.338440 \\
\hline $\mathrm{N}$ & 0.999159 & 3.542641 & 2.517356 \\
\hline C & -0.868697 & 5.197472 & 0.000000 \\
\hline $\mathrm{N}$ & -0.129961 & 4.796333 & 1.183714 \\
\hline
\end{tabular}




\begin{tabular}{|c|c|c|c|}
\hline $\mathrm{N}$ & -0.162194 & -4.029787 & 2.492007 \\
\hline $\mathrm{C}$ & 0.924514 & 4.796361 & 3.101955 \\
\hline $\mathrm{C}$ & 0.347040 & 3.536384 & -1.338440 \\
\hline $\mathrm{C}$ & 0.213325 & 5.591868 & 2.263162 \\
\hline $\mathrm{C}$ & 0.267926 & -3.483882 & 1.338251 \\
\hline $\mathrm{N}$ & -0.129961 & 4.796333 & -1.183714 \\
\hline $\mathrm{N}$ & 0.999159 & 3.542641 & -2.517356 \\
\hline $\mathrm{H}$ & -0.986723 & 6.281232 & 0.000000 \\
\hline $\mathrm{C}$ & 0.267926 & -3.483882 & -1.338251 \\
\hline $\mathrm{N}$ & -0.162194 & -4.029787 & -2.492007 \\
\hline $\mathrm{C}$ & 0.835796 & -4.805160 & 3.060831 \\
\hline $\mathrm{C}$ & 0.213325 & 5.591868 & -2.263162 \\
\hline $\mathrm{C}$ & 0.924514 & 4.796361 & -3.101955 \\
\hline $\mathrm{N}$ & 1.540366 & -3.928826 & 1.183478 \\
\hline $\mathrm{H}$ & 2.559327 & -2.539095 & 0.000000 \\
\hline $\mathrm{C}$ & 1.911875 & -4.748098 & 2.235812 \\
\hline $\mathrm{N}$ & 1.540366 & -3.928826 & -1.183478 \\
\hline $\mathrm{C}$ & 2.316732 & -3.605028 & 0.000000 \\
\hline $\mathrm{C}$ & 0.835796 & -4.805160 & -3.060831 \\
\hline $\mathrm{C}$ & 1.911875 & -4.748098 & -2.235812 \\
\hline $\mathrm{H}$ & 3.236335 & -4.190519 & 0.000000 \\
\hline $\mathrm{H}$ & 0.692908 & -5.330515 & 3.992988 \\
\hline $\mathrm{H}$ & 2.888344 & -5.201637 & 2.312892 \\
\hline $\mathrm{H}$ & 2.888344 & -5.201637 & -2.312892 \\
\hline $\mathrm{H}$ & 0.692908 & -5.330515 & -3.992988 \\
\hline $\mathrm{H}$ & 1.382090 & 5.013657 & 4.055273 \\
\hline $\mathrm{H}$ & -0.079093 & 6.627609 & 2.347298 \\
\hline $\mathrm{H}$ & -0.079093 & 6.627609 & -2.347298 \\
\hline $\mathrm{H}$ & 1.382090 & 5.013657 & -4.055273 \\
\hline $\mathrm{C}$ & 1.760928 & 2.429627 & -3.080332 \\
\hline $\mathrm{H}$ & 1.439230 & 1.508524 & -2.596307 \\
\hline $\mathrm{H}$ & 1.560934 & 2.367647 & -4.151653 \\
\hline $\mathrm{H}$ & 2.829010 & 2.595146 & -2.918434 \\
\hline $\mathrm{C}$ & 1.760928 & 2.429627 & 3.080332 \\
\hline $\mathrm{H}$ & 1.560934 & 2.367647 & 4.151653 \\
\hline $\mathrm{H}$ & 1.439230 & 1.508524 & 2.596307 \\
\hline $\mathrm{H}$ & 2.829010 & 2.595146 & 2.918434 \\
\hline $\mathrm{C}$ & -1.511156 & -3.919661 & 3.043868 \\
\hline $\mathrm{H}$ & -1.445342 & -3.684208 & 4.107918 \\
\hline $\mathrm{H}$ & -2.037449 & -3.118599 & 2.528712 \\
\hline $\mathrm{H}$ & -2.039881 & -4.866739 & 2.911458 \\
\hline
\end{tabular}




$\begin{array}{lrrr}\mathrm{C} & -1.511156 & -3.919661 & -3.043868 \\ \mathrm{H} & -2.037449 & -3.118599 & -2.528712 \\ \mathrm{H} & -1.445342 & -3.684208 & -4.107918 \\ \mathrm{H} & -2.039881 & -4.866739 & -2.911458 \\ \mathrm{C} & -2.261724 & 0.835620 & -2.549992 \\ \mathrm{C} & -2.261724 & 0.835620 & 2.549992 \\ \mathrm{H} & -2.939588 & 0.189082 & -3.108345 \\ \mathrm{H} & -2.867563 & 1.439171 & -1.864925 \\ \mathrm{H} & -1.739813 & 1.514433 & -3.227368 \\ \mathrm{H} & -2.867563 & 1.439171 & 1.864925 \\ \mathrm{H} & -2.939588 & 0.189082 & 3.108345 \\ \mathrm{H} & -1.739813 & 1.514433 & 3.227368\end{array}$

\begin{tabular}{|c|c|c|c|}
\hline atom & $x$ & $y$ & $z$ \\
\hline C & 0.741174 & 0.092689 & 1.760602 \\
\hline O & -0.329950 & -0.444334 & 1.385314 \\
\hline C & 0.741174 & 0.092689 & -1.760602 \\
\hline 0 & 1.153460 & 1.243540 & 1.492353 \\
\hline 0 & -0.329950 & -0.444334 & -1.385314 \\
\hline O & 1.153460 & 1.243540 & -1.492353 \\
\hline $\mathrm{Pd}$ & -0.555562 & -2.069160 & 0.000000 \\
\hline $\mathrm{H}$ & 1.637482 & -4.462832 & 0.000000 \\
\hline $\mathrm{Pd}$ & 0.343148 & 2.526229 & 0.000000 \\
\hline C & -0.699514 & -3.519310 & 1.324099 \\
\hline $\mathrm{N}$ & -1.347171 & -3.582927 & 2.501274 \\
\hline C & 0.705990 & -5.035702 & 0.000000 \\
\hline $\mathrm{N}$ & -0.075560 & -4.712595 & 1.181524 \\
\hline $\mathrm{N}$ & 0.129254 & 4.282543 & 2.483946 \\
\hline C & -1.118828 & -4.808639 & 3.104267 \\
\hline C & -0.699514 & -3.519310 & -1.324099 \\
\hline C & -0.318774 & -5.525626 & 2.274241 \\
\hline C & -0.375178 & 3.802484 & 1.332057 \\
\hline $\mathrm{N}$ & -0.075560 & -4.712595 & -1.181524 \\
\hline $\mathrm{N}$ & -1.347171 & -3.582927 & -2.501274 \\
\hline $\mathrm{H}$ & 0.933383 & -6.101819 & 0.000000 \\
\hline C & -0.375178 & 3.802484 & -1.332057 \\
\hline $\mathrm{N}$ & 0.129254 & 4.282543 & -2.483946 \\
\hline C & -0.760659 & 5.174357 & 3.060691 \\
\hline C & -0.318774 & -5.525626 & -2.274241 \\
\hline C & -1.118828 & -4.808639 & -3.104267 \\
\hline
\end{tabular}




\begin{tabular}{|c|c|c|c|}
\hline $\mathrm{N}$ & -1.580523 & 4.405058 & 1.182652 \\
\hline $\mathrm{H}$ & -2.784607 & 3.167630 & 0.000000 \\
\hline $\mathrm{C}$ & -1.839385 & 5.258633 & 2.241152 \\
\hline $\mathrm{N}$ & -1.580523 & 4.405058 & -1.182652 \\
\hline $\mathrm{C}$ & -2.395837 & 4.189537 & 0.000000 \\
\hline $\mathrm{C}$ & -0.760659 & 5.174357 & -3.060691 \\
\hline C & -1.839385 & 5.258633 & -2.241152 \\
\hline $\mathrm{H}$ & -3.225954 & 4.896147 & 0.000000 \\
\hline $\mathrm{H}$ & -0.548167 & 5.671807 & 3.994985 \\
\hline $\mathrm{H}$ & -2.749947 & 5.832158 & 2.325845 \\
\hline $\mathrm{H}$ & -2.749947 & 5.832158 & -2.325845 \\
\hline $\mathrm{H}$ & -0.548167 & 5.671807 & -3.994985 \\
\hline $\mathrm{H}$ & -1.542967 & -5.063537 & 4.063679 \\
\hline $\mathrm{H}$ & 0.096817 & -6.517003 & 2.372998 \\
\hline $\mathrm{H}$ & 0.096817 & -6.517003 & -2.372998 \\
\hline $\mathrm{H}$ & -1.542967 & -5.063537 & -4.063679 \\
\hline $\mathrm{C}$ & -2.223024 & -2.555510 & -3.059018 \\
\hline $\mathrm{H}$ & -2.064583 & -1.626353 & -2.515000 \\
\hline $\mathrm{H}$ & -1.970473 & -2.402781 & -4.109554 \\
\hline $\mathrm{H}$ & -3.264646 & -2.873798 & -2.971867 \\
\hline C & -2.223024 & -2.555510 & 3.059018 \\
\hline $\mathrm{H}$ & -1.970473 & -2.402781 & 4.109554 \\
\hline $\mathrm{H}$ & -2.064583 & -1.626353 & 2.515000 \\
\hline $\mathrm{H}$ & -3.264646 & -2.873798 & 2.971867 \\
\hline C & 1.456149 & 4.002902 & 3.033602 \\
\hline $\mathrm{H}$ & 1.360900 & 3.752949 & 4.091920 \\
\hline $\mathrm{H}$ & 1.891306 & 3.158298 & 2.504208 \\
\hline $\mathrm{H}$ & 2.090276 & 4.885426 & 2.921323 \\
\hline C & 1.456149 & 4.002902 & -3.033602 \\
\hline $\mathrm{H}$ & 1.891306 & 3.158298 & -2.504208 \\
\hline $\mathrm{H}$ & 1.360900 & 3.752949 & -4.091920 \\
\hline $\mathrm{H}$ & 2.090276 & 4.885426 & -2.921323 \\
\hline $\mathrm{C}$ & 1.676443 & -0.747062 & -2.678104 \\
\hline $\mathrm{C}$ & 1.676443 & -0.747062 & 2.678104 \\
\hline $\mathrm{F}$ & 2.234437 & -1.738965 & -1.948676 \\
\hline $\mathrm{F}$ & 2.651382 & -0.024636 & -3.219659 \\
\hline $\mathrm{F}$ & 0.967059 & -1.317347 & -3.668869 \\
\hline$F$ & 2.234437 & -1.738965 & 1.948676 \\
\hline$F$ & 0.967059 & -1.317347 & 3.668869 \\
\hline $\mathrm{F}$ & 2.651382 & -0.024636 & 3.219659 \\
\hline
\end{tabular}

